\title{
EXAMPLES OF SINGULAR MAXIMAL FUNCTIONS UNBOUNDED ON $L^{p}$
}

\author{
Michael Chist
}

\section{1. $L^{1}$ examples}

In $\mathbb{R}^{2}$ consider a continuous curve $\left\{(t, \gamma(t)): t \in \mathbb{R}^{+}\right\}$, satisfying $\gamma(0)=0$. To it may be associated a maximal function

$$
\mathcal{M}_{\gamma} f(x)=\sup _{r>0} r^{-1} \int_{0}^{r}\left|f\left(x_{1}-t, x_{2}-\gamma(t)\right)\right| d t .
$$

Much labor has been devoted to understanding for which curves $\mathcal{M}_{\gamma}$ is a bounded operator on $L^{p}\left(\mathbf{R}^{2}\right)$. Positive results are known under various geometric hypotheses; see for instance [SW], [CarI], [Carb] and the references therein. In particular, curvature plays a major role, and important contributions to our understanding of its ramifications were made by Rubio de Francia [Carl], [DR], [R].

By analogy with the Calderon-Zygmund theory of singular integrals and the Hardy-Littlewood maximal function, a fundamental question about these more singular operators is what happens for $p=1$, that is, whether $M_{\gamma}$ is of weak type $(1,1)$. No operator of this type, save those which reduce in a very trivial way to the Hardy-Littlewood maximal function in $\mathbb{R}^{1}$ (as when $\gamma \neq 0$ ), has been proved to be of weak type $(1,1)$. For the closest approach to date see [C].

Our first purpose here is to show that in fact the weak $(1,1)$ boundedness fails for a large class of curves, those which are piecewise linear in an appropriate sense. Among these curves are large families for which $L^{p}$ boundedness is already known for all $p>1$. The prototypical example is $\gamma(t)=t^{2}$ for $t=2^{j}$, $j \in \mathbb{Z}$, and $\gamma$ affine on each interval $2^{j} \leq t \leq 2^{j+1}$. The absence of curvature will be essential to our analysis. Rubio de Francia was quite interested in both weak (1,1) boundedness and $L^{p}$ boundedness for operators with singularities along varieties lacking curvature, and again made significant contributions [Carl], [CR].

Our construction will apply in greater generality: Let $\ell_{1}, \ldots \ell_{N}$ be $N$ line segments in $\mathbb{R}^{n}$ of finite length, $n \geq 2$. For cach $j$ let. $\nu_{j} \in S^{n-1}$ be one of the two unit vectors oriented parallel to $\ell_{j}$. Sornewhat abusively we speak of $\nu_{j}$ as the orientation of $\ell_{j}$, and say that $\nu_{i}=\nu_{j}$ if they span the same line in $\nabla^{n}$. Let

Research supported in part by a grant from the National Science Foundation. 
$\lambda_{j}$ denote one-dimensional Hausdorf measure on $\ell_{j}$, normalized to have total mass one. Define the maximal function

$$
M f(x)=\sup _{1 \leq j \leq N} \int_{\ell_{i}}|f(x-y)| d \lambda_{j}(y)
$$

Proposition 1. For each $n \geq 2$ there exists a constant $B_{n}>0$ such that for any $N \geq 1$ and any collection of line segments $\left\{\ell_{j}: 1 \leq j \leq N\right\}$ as above with distinct orientations, the weak type $(1,1)$ operator norm of $M$ is bounded below by $B_{n} N$.

By projecting onto a generic two-dimensional stubspace one secs that $B_{n}$ is actually independent of the dimension, and that it would suffice to prove this for $n=2$. It is not required that the $\ell_{j}$ be in any way related to one another, e.g. that they piece together to form a continuous curve; another situation of some interest is when each has one endpoint at the origin.

Since the operators given by convolution with the $\lambda_{j}$ are uniformly bounded on $L^{1}$, the weak $(1,1)$ operator norm of $M$ is bounded above by $C N$. So the rate of growth is the largest imaginable.

Corollary. Suppose that $\gamma$ is affine in each interval $2^{j} \leq t \leq 2^{j+1}$ and that its derivative takes on infinitely many distinct values. Then $\mathcal{M}_{\gamma}$ is not of weak type $(1,1)$.

It will be apparent from the construction that the same goes for Hilbert transforms along curves.

It is permissible to assume in the proof that each $\ell_{j}$ has length $2^{I(j)}$ for some $I(j) \in Z$. This may be achieved by shrinking each $\ell_{j}$ by a factor between 1 and 2 to a concentric segment with the same orientation; the resulting maximal function is bounded pointwise by twice the original one so it is enough to show that the new one is unbounded.

Suppose that segments $\ell_{1}, \ldots \ell_{N}$ have distinct orientations $\nu_{j}$. By dilating everything by some factor we may suppose without loss of generality that $I(j) \geq 0$ for all $j$. Denote by $\tilde{\ell}_{j}$ the segment $\left\{s \nu_{j}:|s|<8^{-1} 2^{I(j)}\right\}$ centered at the origin. Denote by $\hat{\ell}_{j}$ the segment concentric with $\ell_{j}$ and with the same orientation but half as long.

We wish to construct a set of points $\left\{z_{\alpha}^{k}: 1 \leq k \leq N, 0 \leq \alpha<2^{I(k)}\right\}$ satisfying certain conditions. First some notation, assuming the $z_{\alpha}^{k}$ have already been constructed: Let $\mathcal{A}$ denote the set of sums $w=\sum_{k=1}^{N} z_{\alpha_{k}}^{k}$. Define for each $1 \leq j \leq N$ an equivalence relation on $\mathcal{A}$ as follows. If $w$ is as above, and $w^{\prime}=\sum z_{\beta_{k}}^{k}$, then $w \sim_{j} w^{\prime}$ if and only if $w-w^{\prime}$ is a scalar multiple of $\nu_{j}$. Then the conditions are

(1) $z_{0}^{k}=0$ 
(2) $z_{\alpha}^{k} \in \tilde{\ell}_{k}$

(3) For all $1 \leq j \leq N$ and $w, w^{\prime} \in \mathcal{A}, w \sim_{j} w^{\prime}$ if and only if $\alpha_{i}=\beta_{i}$ for all $i \neq j$.

Implicit in (3) is that for each $k$, the points $z_{\alpha}^{k}$ are distinct if $N>1$. It is quite easy to prove the existence of a set of points $z_{\alpha}^{k}$ satisfying these conditions. Let us just assume it for the moment and proceed with the proof of the proposition.

Let $\epsilon>0$ be a small parameter which will later tend to zero. For each $1 \leq j \leq N, w \in \mathcal{A}$ define

$$
S(w, j, \epsilon)=\left\{x \in \mathbb{R}^{n}: \text { distance }\left(x-\hat{\ell}_{j}, w\right)<\epsilon / 2\right\} \text {. }
$$

Observe that $x \in S(w, j, \epsilon) \Rightarrow$ distance $\left(x-\ell_{j}, w^{\prime}\right)<\epsilon / 2$ for all $w^{\prime} \sim j w$. For $x-\ell_{j}$ is a segment of twice the length of $x-\hat{\ell}_{j}$ with the same center, while by (2) and definition of the equivalence relation, $w^{t}-w=s \nu_{j}$ for some $|s|<4^{-1} 2^{I(j)}$, which is one quarter of the length of $\ell_{j}$.

Let $B(w, \epsilon)$ denote the ball of radius $\epsilon$ centered at $w$ and let $\chi_{E}$ denote the characteristic function of a set $E$. Define

$$
f_{\epsilon}(x)=\sum_{w \in \mathcal{A}} \chi_{B(w, \epsilon)}(x) .
$$

If $w \in \mathcal{A}$ and $x \in S(w, j, \epsilon)$ then by the observation of the last paragraph, the segment $x-\ell_{j}$ meets $B\left(w^{\prime}, \epsilon\right)$ in a set of length at least $\epsilon / 2$, for evcry $w^{\prime} \sim j w$. There are $2^{I(j)}$ such $w^{\prime}$. On the other hand $x-\ell_{j}$ has length $2^{I(j)}$. Consequently

$$
\begin{aligned}
x \in S(w, j, \epsilon) & \Rightarrow \int f_{\epsilon}(x-y) d \lambda_{\jmath}(y) \geq \epsilon / 2 \\
& \Rightarrow M f_{\epsilon}(x) \geq \epsilon / 2 .
\end{aligned}
$$

So our task is to show that the measure of $\cup_{w, j} S(w, j, \epsilon)$ is large. First consider the union over $w \in \mathcal{A}$ with $j$ fixed. To each $w$ associate a canonical representative $\hat{w}$ of its $j$-th equivalence class, specified by $\hat{w}=\sum z_{\alpha_{k}}^{k}$ with $\alpha_{j}=0$. The set of all $\hat{w}$ has cardinality $|\mathcal{A}| / 2^{\prime(j)}$. We maintain that if $\varepsilon$ is chosen suffeiently small, depending on $N,\left\{\ell_{j}\right\}$, and on $\mathcal{A}$, the sets $S(\hat{w}, j, \epsilon)$ will be disjoint for any two such $\hat{w}$. Indeed $S(\hat{w}, j, \epsilon)=\left\{x: \operatorname{distance}\left(x, \hat{w}+\hat{\ell}_{j}\right)<\right.$ $\epsilon / 2\}$. If these intersect for $\hat{w}_{1}, \hat{w}_{2}$ then distance $\left(\hat{w}_{1}-\hat{w}_{2}, \hat{\ell}_{j}-\hat{\ell}_{j}\right)<\epsilon$. But $\hat{\ell}_{j}-\hat{\ell}_{j} \subset \mathbb{R} \nu_{j}$, and $\hat{w}_{1}-\hat{w}_{2} \in \mathbb{R} \nu_{j}$ means $\hat{w}_{1} \sim, \hat{w}_{2}$, which by (3) implies $\hat{w}_{1}=\hat{w}_{2}$. So once $\epsilon$ is sufficiently small, distance $\left(\hat{w}_{1}-\hat{w}_{2}, \hat{\ell}_{j}-\hat{\ell}_{j}\right)>\epsilon$. Since $A$ is a finite set, some $\epsilon>0$ suffices to make all these sets disjoint for each $j$. So

$$
\begin{aligned}
\left|\bigcup_{w \in \mathcal{A}} S(w, j, \epsilon)\right| & \geq\left|\bigcup_{\dot{w}} S(\hat{w}, j, \epsilon)\right| \\
& =\sum_{\dot{w}}|S(\hat{w}, j, \epsilon)| \\
& \geq c_{1} \epsilon^{n-1} 2^{I(j)} \cdot 2^{-I(j)}|\mathcal{A}|
\end{aligned}
$$


where $c_{1}$ is an absolute constant depending only on the dimension of the ambient space. The first factor of $2^{I(j)}$ represents the length of $\ell_{j}$, while $2^{-I(j)}|\mathcal{A}|$ is the cardinality of the set of all $j$-th equivalence classes.

The final point is that for distinct $j$ 's, the sets $S(w, j, \epsilon)$ are also essentially disjoint. Indeed if $i \neq j$ and if $w, w^{\prime} \in \mathcal{A}$ (possibly equal) then $\mid S(w, j, \epsilon) \cap$ $S\left(w^{t}, i, \epsilon\right) \mid=O\left(\epsilon^{n}\right)$ as $\epsilon \rightarrow 0$ since $\nu_{j} \neq \nu_{i}$; we have intersecting tubular neighborhoods of two line segments with differing orientations. Therefore

$$
\left|\bigcup_{\substack{1 \leq j \leq N \\ w \in \mathcal{A}}} S(w, j, \epsilon)\right| \geq c_{1} \epsilon^{n-1} N|\mathcal{A}|-O\left(\epsilon^{n}\right) .
$$

The constant implied in the error term $O\left(\epsilon^{\mathfrak{n}}\right)$ depends on everything in the hypotheses and construction and is in no way under control. However by taking $\epsilon$ sufficiently small we obtain

$$
\left|\bigcup_{\substack{1 \leq j \leq N \\ w \in \mathcal{A}}} S(w, j, \epsilon)\right| \geq \frac{c_{t}}{2} \epsilon^{n-1} N|\mathcal{A}| .
$$

Now $\left\|f_{\epsilon}\right\|_{L^{1}}=c_{2}|\mathcal{A}| \epsilon^{n}$. Therefore

$$
\begin{aligned}
\left|\left\{x: M f_{c}(x)>\epsilon / 2\right\}\right| & \geq \frac{c_{1}}{2} \epsilon^{n-1} N|\mathcal{A}| \\
& =c_{3} N \epsilon^{-1}\left\|f_{\epsilon}\right\|_{L^{1}}
\end{aligned}
$$

where $c_{2}, c_{3}$ are absolute constants.

This construction is completely inapplicable to curves such as $\gamma(t)=t^{2}$ with nonvanishing curvature. The question of their $L^{1}$ behavior remains open.

It remains only to prove the existence of points $z_{\alpha}^{k}$ satisfying conditions (1) through (3). We proceed by induction on $k$. We claim that for each $1 \leq k \leq N$ it is possible to choose $\left\{z_{\alpha}^{i}: i \leq k, \alpha<2^{I(i)}\right\}$ so that $z_{0}^{i}=0, z_{\alpha}^{i} \in \tilde{\ell}_{i}$, and so that (3) holds for all $j \leq N$ but only for $w, w^{t}$ already defined, that is of the form $w=\sum_{i \leq k} z_{\alpha_{i}}^{i}$ and similarly for $w^{\prime}$. At step $k=1(3)$ is no constraint. Suppose the step $k$ is done. For $k+1, z_{0}^{k+1}$ must be the origin. $z_{1}^{k+1}$ is permitted to be any point of $\tilde{\ell}_{k+1}$ which is not an element of any of the finitely many lines $\sum_{i \leq k}\left(z_{\alpha_{i}}^{i}-z_{\beta_{j}}^{i}\right)+R \cdot \nu_{j}, 1 \leq j \leq N, j \neq k+1$. (The case $j=k+1$ is already handled by the induction process.) But since $\nu_{j}$ and $\nu_{k+1}$ are distinct, only finitely many points of $\tilde{\ell}_{k+1}$ violate this constraint. Choose $z_{1}^{k+1}$ to be any point of $\tilde{\ell}_{k+1}$ which is not on one of the forbidden lines. Now consider $z_{2}^{k+1}$. The only forbidden values are those for which one of $z_{2}^{k+1}-z_{1}^{k+1}, z_{2}^{k+1}-z_{0}^{k+1}$ lies on one of the forbidden lines. So again only finitely many points of $\tilde{\ell}_{k+1}$ are forbidden and there is no problem in selecting $z_{2}^{k+1}$. Continuing in this way we obtain all the required $z_{\alpha}^{k+1}, \alpha<2^{I(k+1)}$. 


\section{2. $L^{p}$ examples}

Until now it has been unknown whether there are any curves for which $\mathcal{M}_{\gamma}$ is bounded on $L^{p}$ for some but not all $p \in(1, \infty)$. What one can deduce without computation from the construction above is that for any infinite sequence $\left\{\ell_{j}\right\}$ with distinct orientations and lengths tending to zero, there exists an Orlicz class which is locally more restrictive than $L^{1}$, and which is not mapped to weak $L^{1}$ (locally) by the maximal function. Suppose we were to attempt to apply the same argument to obtain an $L^{p}$ counterexample for some $p>1$, in $\boldsymbol{R}^{2}$. For any fixed distribution of points $w,\left\|f_{\epsilon}\right\|_{p} \approx \epsilon^{2 / p}$ while $\left\|M f_{\varepsilon}\right\|_{p} \lesssim \epsilon^{1+p^{-1}} \ll \epsilon^{2 / p}$ as $\epsilon \rightarrow 0$. Therefore a counterexample can no longer be obtained by passing to the limit. Since that limit allowed us to avoid the intricate question of how the sets $S(w, j, \epsilon)$ overlap, a close examination of the dependence of this overlapping on $\epsilon$, on $p$ and on $\mathcal{A}$ is required for $p>1$.

We shall not attempt such an analysis. Instead we shall indicate how in certain particular cases a different choice of the "lattice" $A$ leads to a very computable situation and to $L^{p}$ counterexamples for certain $p>1$.

First let $N$ be a large integer and for each $1 \leq j \leq N$, let $\ell_{j}$ be the segment in $\mathbb{R}^{2}$ centered at the origin, with slope $j / N$ and length $2^{j+1}$. Let

$$
M_{N} f(x)=\sup _{1 \leq j \leq N}\left|f * \mu_{j}(x)\right|
$$

where $\mu_{j}$ is arclength measure on $\ell_{j}$, normalized to be a probability measure.

Proposition 2. For each $p<3 / 2$ the operator norm of $M_{N}$ on $L^{p}$ is $\gtrsim$ $N^{(3 / p)-2}$, hence tends to $\infty$, as $N \rightarrow \infty$.

For $p \geq 2, M_{N}$ remains uniformly in $L^{p}$. This follows either from the arguments of [CW], from Fourier transform and square function estimates, or from the method of covering lemmas as developed by Córdoba and $R$. Fefferman. Naturally it would be most interesting to resolve matters in the range $3 / 2 \leq p<2$.

To prove the proposition let

$$
\mathcal{A}=\left\{(x, y) \in \mathbf{Z} \times\left(N^{-1} \mathbf{Z}\right): 0 \leq x, y \leq 2^{N}\right\} .
$$

Here $N^{-1} \mathbf{Z}=\{i / N: i \in \mathbf{Z}\}$. Let $f_{\epsilon}$ be the sum of characteristic functions of all balls of radius $\epsilon$ centered at points of $\mathcal{A}$. Any line of slope $k / N$, with $1 \leq k \leq N$, which passes through some one of these balls, must pass through $\geq 2^{N}$ of them, unless the first-mentioned ball is located near an edge of the configuration. In fact the balls it meets will be equally spaced along it, with spacing interval between 1 and $\sqrt{ } 2$. Therefore $f_{\epsilon} * \mu_{j}$ is $\gtrsim \epsilon$ on a union of about $N 2^{N}$ disjoint strips, each of width $\approx \epsilon$ and length $\approx 2^{N}$. 
Unfortunately these strips overlap substantially when $j$ is varied. We wish to choose $\epsilon$ sufficiently small that for any one of the strips, the measure of the union of its intersections with all other strips does not exceed half its measure. Then the measure of the union of all the strips is comparable to the sum of their measures, which is readily computable.

Fix a strip with slope $j / N$ and consider its intersection with a strip of different slope $k / N$. The measure of the intersection is $\leq \epsilon^{2} /\left|\frac{j}{N}-\frac{k}{N}\right|$. Now consider all strips with this slope $k / N$ which meet the fixed strip. There are $\lesssim N 2^{N}\left|\frac{j}{N}-\frac{k}{N}\right|$ of them. Indeed the given strip passes within distance $\epsilon$ of some point $(0, m / N)$ with $m \in \mathbf{Z}$, and each strip with slope $k / N$ passes near a unique point $(0, n / N)$. However by plane geometry, only those for which $\left|\frac{j}{N}-\frac{k}{N}\right| 2^{N} \geq|n-m| / N$ can cross the fixed strip, since it has length $\leq 2^{N}$. The number of allowed values of $n$ is $\lesssim N 2^{N}\left|\frac{j}{N}-\frac{k}{N}\right|$ as claimed.

Therefore the sum of the measures of the intersections with all strips with slope $k / N$ which meet the given strip is $\leqq \epsilon^{2} N 2^{N}$. Summing over all $1 \leq k \leq N$, we find that the measure of the union of the intersections of the fixed strip with all others is $\leqslant \epsilon^{2} N^{2} 2^{N}$. Since the given strip has measure $\approx \epsilon 2^{N}$, we require

$$
\epsilon=c_{0} N^{-2}
$$

where $c_{0}$ is a small constant.

Supposing this, the measure of the union of all strips of all slopes is comparable to the sum of their measures, which is $\gtrsim N \cdot N 2^{N} \cdot \epsilon 2^{N}$, the product of the number of slopes, the number of strips of a given stope, and the measure of one strip. Since $M_{N} f_{\epsilon} \gtrsim \epsilon$ on this set,

$$
\begin{aligned}
\frac{\left\|M_{N} f_{\epsilon}\right\|_{p}^{p}}{\left\|f_{\epsilon}\right\|_{p}^{p}} & \geq \frac{\epsilon^{p} \cdot \epsilon N^{2} 2^{2 N}}{\epsilon^{2} N 2^{2 N}} \\
& =\epsilon^{p-1} N \\
& =c_{0}^{p-1} N^{3-2 p} .
\end{aligned}
$$

This establishes Proposition 2.

If each of the slopes is perturbed by an amount $\delta_{j}$, the argument remains valid if $2^{N} \delta_{j} \leq \epsilon=c_{0} N^{-2}$ for $j \approx N$. Thus $\delta_{j} \leq 2^{-N} N^{-2}$; the example is extremely sensitive to perturbations. We don't know whether this reflects a weakness in the construction or a gentine feature of the problem.

Another class of examples may be obtained by taking $\ell_{j}$ to have slope $j^{-r}$ and length $2^{j}, j \geq 1$. Let $M^{(r)}$ be the associated maximal function.

Proposition 3. $M^{(r)}$ is unbounded on $L^{p}$ for all $p<(2 r+2) /(2 r+1)$.

This time let $N$ be large and take

$$
\mathcal{A}=\left\{(x, y) \in \mathbf{Z} \times \mathbf{Z}: 0 \leq x, y \leq 2^{N}\right\}
$$


As always let $f_{\epsilon}$ be the sum of characteristic functions of $\epsilon$-balls centered at the points of $\mathcal{A}$. If a line of slope $j^{-r}$ passes through a point of $\mathcal{A}$, then typically it will pass through about $N^{-r} 2^{N}$ points of $\mathcal{A}$. These are equally spaced along the line, with spacing $\approx j^{r}$. We restrict attention to the range $N / 2<j \leq N$. Then $M^{(r)} f_{\epsilon} \gtrsim \epsilon N^{-r}$ on the union of the strips. There are about $N^{r} 2^{N}$ strips of slope $j^{-r}$ for each $j$ (for now the midline of such a strip crosses the $y$-axis at a point $\left(0, n j^{-r}\right)$ where $\left.0 \leq n \leq j^{r} 2^{N}\right)$, for a total of about $N^{r+1} 2^{N}$ strips. Each has area $\approx \epsilon 2^{N}$.

Two strips, with slopes $j^{-r}$ and $k^{-r}$, meet in a set of area $\lesssim \epsilon^{2} /\left|j^{-r}-k^{-r}\right|$. If $j$ is fixed, the total of number of strips with any particular slope $k^{-r}$ which meet it is $\lesssim N^{r} 2^{N}\left|j^{-r}-k^{-r}\right|$. Thus the measure of the union of the intersection of one strip with all other strips of any particular slope is $\leqslant \epsilon^{2} N^{r} 2^{N}$, and with all other strips of all slopes $(k \approx N)$ is $\leqslant \epsilon^{2} N^{r+1} 2^{N}$. Therefore the overlapping condition should be $\epsilon^{2} N^{r+1} 2^{N}=c_{0} \in 2^{N}$, or

$$
\epsilon=c_{0} N^{-r-1} \text {. }
$$

Then $M^{(r)} f_{\epsilon} \gtrsim \epsilon N^{-r}$ on a set of measure $\approx N \cdot N^{r} 2^{N} \cdot \epsilon 2^{N}$, whence

$$
\begin{aligned}
\frac{\left\|M^{(r)} f_{\epsilon}\right\|_{p}^{p}}{\left\|f_{\epsilon}\right\|_{p}^{p}} & \geqslant \frac{\epsilon^{p} N^{-r_{p}} \cdot \epsilon N^{r+1} 2^{2 N}}{\epsilon^{2} 2^{2 N}} \\
& =\epsilon^{p-1} N^{r+1-r p} \\
& =c_{0}^{p-1} N^{-(p-1)(r+1)} N^{r+1-r_{p}} \\
& =c_{0}^{p-1} N^{(2 r+2)-(2 r+1) p} .
\end{aligned}
$$

If $p<(2 r+2) /(2 r+1)$, this blows up as $N \rightarrow \infty$.

Though we have assumed the $\ell_{j}$ to be centered at the origin, the location of their centers actually is of no consequence in this construction. Translating $\ell_{j}$ by $a_{j}$ has the effect of translating the whole family of strips with the same orientation as $\ell_{j}$ by $a_{j}$. The upper bounds on intersections remain valid independent of the transiations, so the computations are unaffected.

For some families of translations, much simpler examples can be used to show the unboundedness on $L^{p}$. Roughly speaking this happens if the segments are translated by distances which are sufficiently large compared to their lengths. The best case occurs when all the $\ell_{j}$ are centered at the origin; if the maximal function $M_{3}$ associated to a particular family of segments $\ell_{j}$ with centers $a_{j}$ is bounded on $L^{p}$, then so is the maximal function $M_{2}$ obtained when all the $\ell_{j}$ are translated so as to be centered at the origin. For $M_{2}$ is majorized by a constant multiple of the composition of $M_{1}$ with $\tilde{M}_{1}$ where $\tilde{M}_{1}$ is obtained from $M_{1}$ by conjugating with refection about the origin.

Let $\gamma: \mathbb{R} \mapsto \mathbb{R}$ be continuous, odd, and convex for $t>0$. Consider the "Hilbert transform"

$$
H_{\gamma} f(x)=\int_{-\infty}^{\infty} f\left(x_{1}-t, x_{2}-\gamma(t)\right) \frac{d t}{t} .
$$


For such a $\gamma$, there is a necessary and sufficient condition for $H_{\gamma}$ to be bounded on $L^{2}\left(\mathbb{R}^{2}\right)[\mathbf{N}]$. It is that the auxiliary quantity $h(t)=t \gamma^{\prime}(t)-\gamma(t)$ should have bounded doubling time, that is, there should exist $C<\infty$ such that for every $t>0, h(C t) \geq 2 h(t)$. This condition is further known to guarantee $L^{p}$ boundedness of the maximal function $M_{\gamma}$ for all $p \geq 2$. (However for the maximal function, the condition is far from necessary.) More recentiy it has been shown [Carb] that if $h$ satisfies an infinitesimal version of the doubling condition, $h^{\prime}(t) \geq \epsilon h(t) / t$ for some $\epsilon>0$, then $M_{\gamma}$ and $H_{\gamma}$ are bounded on $L^{p}$ for all $1<p<\infty$. However it was also demonstrated in [Carb] that if $\gamma\left(2^{j}\right)=j 2^{j}$ for $j \in \mathbb{Z}$ and $\gamma$ is defined to be affine in the intervening intervals, then $H_{\gamma}$ is not bounded on $L^{p}$ for any $p \neq 2$. This was the first example in which $H_{\gamma}$ was known to be bounded on $L^{2}$ but not on all $L^{p}, 1<p<\infty$.

As a final example of the method we have:

Proposition 4. Let $\gamma\left(2^{j}\right)=j 2^{j}$ for $j \in \mathbf{Z}$, and let $\gamma$ be affine in each interval $\left[2^{j}, 2^{j+1}\right]$. Then $M_{\gamma}$ is unbounded on $L^{p}$ for all $p<4 / 3$.

Again it would be of substantial interest to know what is the right range of $p$.

This curve is the union of segments $\ell_{j}=\left\{(t, \gamma(t)): 2^{j} \leq t \leq 2^{j+1}\right\} . \ell_{j}$ has slope $j+2$. Let $\mu_{j}$ be the associated probability measure. Then $M_{\gamma}$ is bounded pointwise by twice

$$
M f(x)=\sup _{j}|f| * \mu_{j}
$$

Once again let $N$ be large and consider the maximum over all $j, N / 2<$ $j \leq N$. To make this look more like our last example introduce reflected segments $\tilde{\ell}_{j}$, where $\left(x_{1}, x_{2}\right)^{\sim}=\left(x_{2}, x_{1}\right)$. This transformation commutes with translation, so the maximal function has the same boundedness properties as $\tilde{M} f=\sup |f| * \tilde{\mu}_{j}$, where $\tilde{\mu}_{j}$ denotes normalized arclength measure on $\tilde{\ell}_{j}$. Then $\tilde{\ell}_{j}$ has length $\approx j 2^{j}$, and slope $(j+2)^{-1}$. For $j \approx N$, the length is $\approx N 2^{j}$. If we dilate by a factor of $N^{-1}$ (in both coordinates), we encounter a family of segments with slopes $(j+2)^{-1}$ and lengths $\approx 2^{j}$, for $j \approx N$. The computations of the last proposition, for $r=1$, now apply verbatim.

\section{Dependence on $p$}

Although we have seen that the $L^{p}$ boundedness of our singular maximal functions does depend on $p$, one might hold out hope that a relatively simple situation prevails, that for any given curve boundedness either holds only for $p \geq 2$, or for all $p>1$. This is however not the case for

$$
M_{\gamma} f(x)=\sup _{r>0} r^{-1} \int_{0}^{r}\left|f\left(x_{1}-t, x_{2}-\gamma(t)\right)\right| d t
$$


Proposition 5. For each $p>1$ there exists $q \in(1, p)$ and a continuous, convex curve $\{(t, \gamma(t)): t \geq 0\}$ with $\gamma(0)=0$ and $\gamma$ piecewise $C^{\infty}$, such that $M_{\gamma}$ is bounded on $L^{p}$ but unbounded on $L^{q}$.

Proof: To construct $\gamma$, define $\gamma(t)=t \log t$ for $0<t \leq 1$, where the logarithm is to the base 2 . For each even integer $j \geq 0$ let $\delta_{j} \in\left(0, \frac{1}{2}\right]$ be a number to be specified later and set

$$
\gamma(t)=j 2^{j}+(j+2) \cdot\left(t-2^{j}\right) \quad \text { for } t \in I_{j}
$$

where

$$
I_{j}=\left[2^{j},\left(1+\delta_{j}\right) 2^{j}\right]
$$

Thus the curve agrees with the example in Proposition 4 on the union of the $I_{j}$. Set $J_{j}=\left[2^{j}, 2^{j+2}\right] \backslash I_{j}$ for even, nonnegative $j$. On each $J_{j}$ construct the unique function $\gamma$ which is continuous, has constant second derivative, agrees with the values already set at the endpoints, and is $C^{1}$ at $t=\left(1+\delta_{j}\right) 2^{j}$. Now $\gamma(t)$ is defined for all $t>0$. It is continuous at 0 , convex, and piecewise $C^{\infty}$.

Define the maximal functions

$$
\begin{aligned}
M_{0} f(x) & =\sup _{0<r \leq t} r^{-1} \int_{0}^{r}\left|f\left(x_{1}-t, x_{2}-\gamma(t)\right)\right| d t, \\
M_{\text {good }} f(x) & =\sup _{j \geq 0} 2^{-j} \int_{J_{j}}\left|f\left(x_{1}-t, x_{2}-\gamma(t)\right)\right| d t, \\
M_{\text {bad }} f(x) & =\sup _{j \geq 0} 2^{-j} \int_{I_{j}}\left|f\left(x_{1}-t, x_{2}-\gamma(t)\right)\right| d t
\end{aligned}
$$

where the last two suprema are taken over all even integers $j \geq 0$. Then

$$
M_{\gamma} \leq M_{0}+2 M_{\text {good }}+2 M_{\mathrm{bad}}
$$

and for $f \geq 0$,

$$
M_{\gamma} f \geq \frac{1}{2} M_{\text {bad }} f
$$

The first inequality will be used to show that $M_{\gamma}$ is bounded on $L^{p}$, the second to show that it is unbounded on $L^{q}$.

First of all for $t \leq 1$, the curve is homogeneous in the sense of Stein and Weiss [SW], so $M_{0}$ is bounded on $L^{p}$ for all $p>1$. For $M_{\text {good note that }} h(t)=$ $t \gamma^{\prime}(t)-\gamma(t)$ satisfies $h(4 t) \geq c_{0} h(t)$ for all $t \geq 1$ where $c_{0}>1, h^{\prime}(t) \geq \eta h(t) / t$ for some $\eta>0$, and each $J_{j} \subset\left[2^{j}, 2^{j+2}\right]$ is an interval. Under these conditions the analysis of [Carb] applies without modification to the disconnected curve $\left\{(t, \gamma(t)): t \in \cup I_{j}\right\}$ to prove that $M_{\text {good }}$ is also bounded on $L^{p}$ for all $p>1$.

So everything turns on the properties of $M_{\text {bad }}$. To make it bounded on $L^{p}$ let us require that

$$
\sum_{j=0}^{\infty} \delta_{j}^{p}<\infty
$$


Define measures $\mu_{j}$ by

$$
\int f d \mu_{j}=2^{-j} \int_{I_{j}} f(t, \gamma(t)) d t
$$

Their total variations satisfy $\left\|\mu_{j}\right\|=\delta_{j}$ and for all $f$,

$$
M_{\text {bad }} f \leq\left(\sum_{j=0}^{\infty}\left(|f| * \mu_{j}\right)^{p}\right)^{1 / p} .
$$

Thus

$$
\begin{aligned}
\left\|M_{\mathrm{bad}} f\right\|_{p}^{p} & \leq \sum\left\||f| * \mu_{j}\right\|_{p}^{p} \\
& \leq\|f\|_{p}^{p} \sum \delta_{j}^{p} .
\end{aligned}
$$

Thus $M_{\text {bad }}$ will be bounded on $L^{p}$ provided the $\delta_{j}$ are chosen so that (*) holds. For simplicity let us make $\delta_{j}$ depend only on $N$ on each interval $2^{N} \leq j<2^{N+1}$, say $\delta_{j}=\epsilon_{N}$. Then $(*)$ becomes

$$
\sum_{N=0}^{\infty} 2^{N} \epsilon_{N}^{p}<\infty
$$

To derive a necessary condition for $L^{q}$ boundedness set

$$
M^{N} f=\sup _{2^{N} \leq j<2^{N+1}}|f| * \mu_{j} .
$$

By dilating by a factor of $\epsilon_{N}^{-1}$ and using the fact that $\left\|\mu_{j}\right\|=\epsilon_{N}$, we see from Propositions 4 and 3 (with $r=1$ ) that the $L^{g}$ operator norm of $M^{N}$ is bounded below by

$$
C_{q} 2^{N\left(\frac{4}{q}-3\right)} \epsilon_{N} .
$$

Fix an exponent $r \in(1, p)$ and set

$$
\epsilon_{N}=2^{-N / r} .
$$

Then $(* *)$ is satisfied. However

$$
2^{N\left(\frac{4}{q}-3\right)} \epsilon_{N} \rightarrow \infty
$$

as $N \rightarrow \infty$ provided $q$ is chosen sufficiently close to 1 , depending on $r$. Therefore the $M^{N}$ are not uniformly bounded in $L^{q}$, so $M_{\text {bad }}$ is unbounded.

In this computation it is important that the lower bound for the rate of growth with $N$ in Proposition 3 tends to the optimal rate as $p \rightarrow 1^{+}$, even though it may not be optimal for any $p>1$.

The proof of Proposition 5 was inspired by a conversation with Roger Jones. 


\title{
References
}

[Carb] A. Carbery, M. Christ, J. Vance, S. Wainger and D. WatSON, Operators associated to flat plane curves: $L^{p}$ estimates via dilation methods, Duke Math. J. (to appear).

[Carl] H. Carlsson, A. Córdoba, M. Christ, J. DuoandikoetXea, J.L. Rubio DE Francia, J. VANCE, S. Wainger and D. Weingerg, $L^{p}$ estimates for maximal functions and Hilbert transforms along flat convex curves in $\mathbb{R}^{2}$, Bulletin AMS 14 (1986), 263-267.

[CW] H. CaRlsSON AND S. WAINGER, Maximal functions related to convex polygonal lines, Indiana Univ. Math. J. 34 (1985), 815-823.

[C] M. CHRIST, Weak type $(1,1)$ bounds for rough operators, Annols of Math 128 (1988), 19-42.

[CR] M. Christ and J. L. Rubio de Francia, Weak type $(1,1)$ bounds for rough operators, II, Invent. Math. 93 (1988), 225-237.

[DR] J. DuoAndikoetxea AND J. L. RubIo de Francia, Maximal and singular integral operators via Fourier transform estimates, Invent. Math. $84(1986), 541-561$.

[N] A. Nagel, J. Vance, S. Wainger and D. Weingerg, Hilbert transforms for convex curves, Duke Math. J. 50 (1983), 735-744.

[R] J. L. Rubio DE Francia, Maximal functions and Fourier transforms, Duke Math. J. 53 (1986), 395-404.

[SW] E. M. STEIN AND S. WAINGER, Problems in harmonic analysis related to curvature, Bull. AMS 84 (1978), 1239-1295.

\author{
Department of Mathematics \\ University of California at Los Angeles \\ Los Angeles, California 90024 \\ U.S.A.
}

